Document downloaded from:

http://hdl.handle.net/10251/52672

This paper must be cited as:

Rodríguez Fortuño, FJ.; Marino, G.; Ginzburg, P.; O'connor, D.; Wurtz, GA.; Martínez Abietar, AJ.; Zayats, AV. (2013). Near-Field Interference for the Unidirectional Excitation of Electromagnetic Guided Modes. Science. 340(6130):328-330. doi:10.1126/science.1233739.

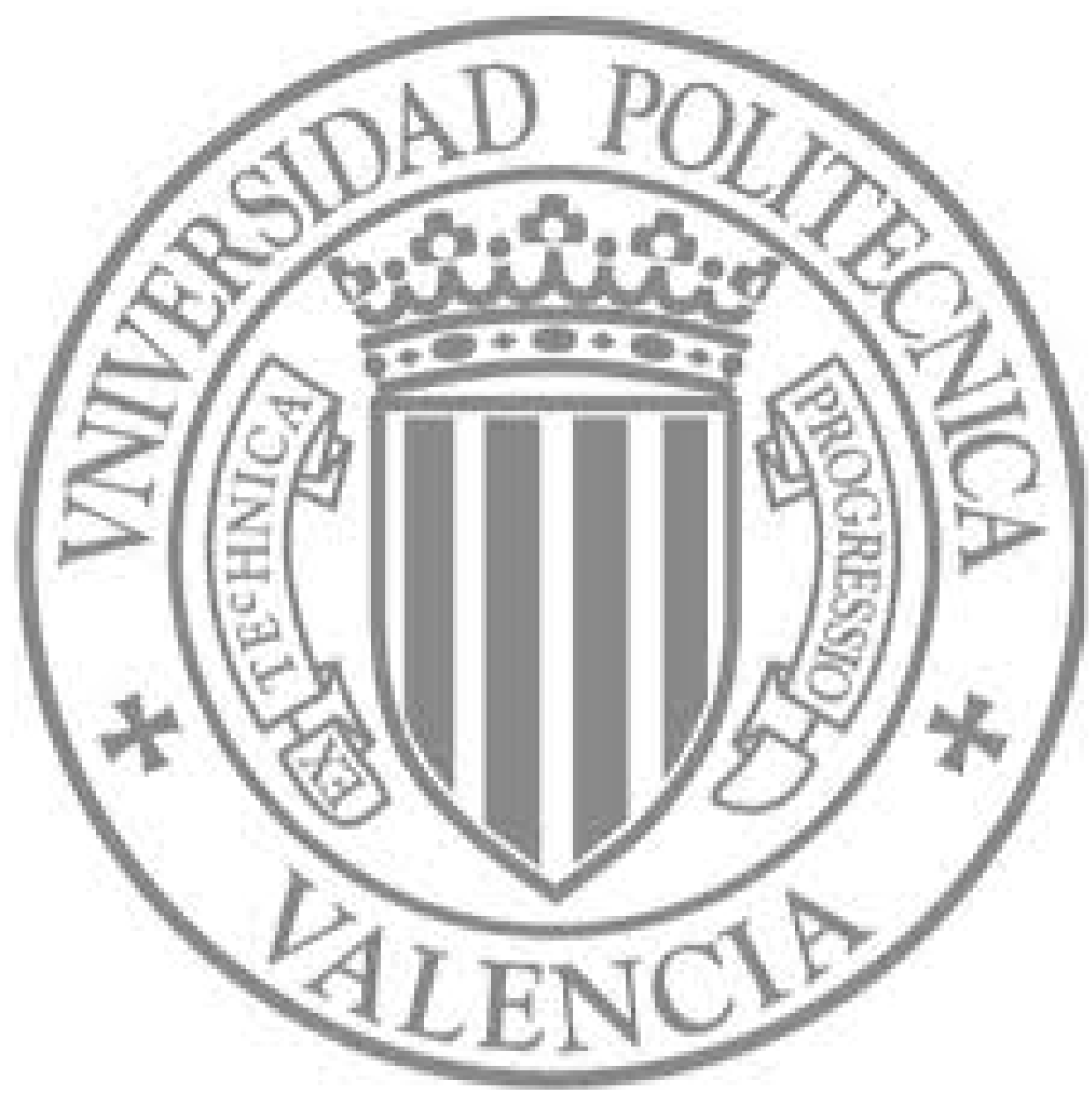

The final publication is available at

http://dx.doi.org/10.1126/science.1233739

Copyright American Association for the Advancement of Science 


\title{
Near field interference for the unidirectional excitation of electromagnetic guided modes
}

\author{
Francisco J. Rodríguez-Fortuño ${ }^{1,2}$, Giuseppe Marino ${ }^{1}$, Pavel Ginzburg ${ }^{1}$, Daniel O'Connor ${ }^{1}$, \\ Alejandro Martínez ${ }^{2}$, Gregory A. Wurtz ${ }^{1}$ and Anatoly V. Zayats ${ }^{1}$ \\ ${ }^{1}$ Department of Physics, King's College London, Strand, London WC2R 2LS, United Kingdom \\ ${ }^{2}$ Nanophotonics Technology Center, Universitat Politècnica de València, Valencia 46022, Spain
}

Wave interference is a fundamental manifestation of the superposition principle with numerous applications. While in conventional optics interference occurs between waves undergoing different phase advances during propagation, we show that the vectorial structure of the near-field of an emitter is essential for controlling its radiation as it interferes with itself on interaction with a mediating object. We demonstrate that the near field interference of a circularly polarized dipole results in the unidirectional excitation of guided electromagnetic modes in the near-field, with no preferred far-field radiation direction. By mimicking the dipole with a single illuminated slit in a gold film, we measured unidirectional surface-plasmon excitation in a spatially symmetric structure. The surface wave direction is switchable with the polarization.

Interference is the cornerstone of various phenomena in nature enabling numerous applications. In optics, it is intensively used in microscopy, stellar measurements, spectroscopy, and communication technologies, among many others, and is the basis behind the concepts of reflection, refraction and light bending $(1,2)$. Typically, interference occurs due to the relative phase lag of different propagating waves. On the other hand, nanophotonics -the branch of optics studying the interaction of light with subwavelength nanoscale structures- deals inherently with phenomena that occur via near-field interactions before appreciable phase lags can be accumulated (3). A radiationless form of interference in the near field (4) is behind new exciting applications such as the focusing of evanescent components to achieve subwavelength resolution in imaging (5-8). Near field interference achieved through the full coherent control of the phase and amplitude of excitation light allows asymmetric spatial field localization $(9,10)$ and selection of propagation paths at intersections of waveguides (11).

We demonstrate near field interference by considering a single source of radiation coupled to a mode with a vectorial structure of electromagnetic field. Using an additional degree of freedom provided by the vectorial character of the field, control over the near-field interference can be achieved. We show that an elliptically polarized dipole can produce destructive or constructive interference of different evanescent components in its near field, and as a result, excite electromagnetic modes in neighbouring material structures, such as dielectric and plasmonic waveguides and diffraction gratings, with a controlled directionality of propagation. 

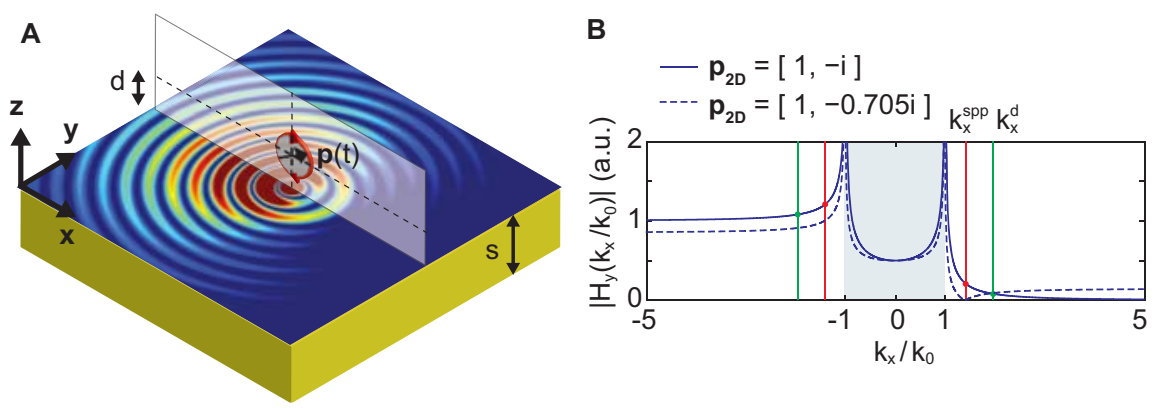

Figure 1. (A) Schematics of the circularly polarized dipole over a planar waveguiding structure (metal-dielectric interface). A cross-section of the calculated magnetic field distribution is overlaid at the surface of a metal $(\varepsilon=-2+0.1 \mathrm{i}, \mathrm{d}=0.3 \lambda)$, showing the asymmetric excitation of SPPs. (B) Spatial frequency spectra of a circularly polarized (continuous line) and elliptically polarized (dashed line) dipole.

Recently, several solutions for the directional excitation of surface plasmon polariton (SPP) waves $(12,13)$ have been proposed, including a backside illuminated slit near a surface Bragg grating (14), the use of asymmetric slanted gratings (15) and chirped gratings (16), tilted angle illumination of slits and gratings $(17,18)$, or the illumination of nearby compact nanoantennas (19). Directional extraction of light from localized emitters with nanoantennas (20) has been demonstrated. All of the above approaches have a resonant nature and rely on the careful selection of the wavelength and geometrical parameters of the structure, with the direction of excitation determined by an asymmetry of the structure and/or the incident light direction. The directional coupling to planar dielectric photonic waveguides is typically achieved with diffraction gratings (21) carefully designed for a specific direction of excitation. Near field dipolar interference provides a fundamentally different approach to unidirectional guided wave excitation with broadband (nonresonant) characteristics and the opportunity to achieve polarization-tuneable directionality.

The phenomenon of unidirectional excitation can be understood by considering a dipole placed at a subwavelength distance $d$ from a waveguide surface, for example a dielectric slab or a single metal-dielectric interface (Fig. 1A). The high spatial frequency components of the dipole radiation allow the excitation of guided modes in the waveguide. The fundamental principle behind the effect of unidirectionality is the selective vectorial excitation of the electrical field of the waveguide mode, depending on the emitted polarization. At the location of the waveguide immediately below the dipole, the vertically oriented component of the dipole is coupled to the transverse field of the electromagnetic mode, while the horizontal component of the dipole drives the longitudinal component. Both components have different amplitude and phase. A properly chosen polarization of the dipole will result in destructive interference in one prescribed direction and a mode propagating in the opposite direction.

Without restriction on the nature of waveguided modes, we consider a rigorous quantitative description based on phase-matching conditions between the source dipole and the structure's modes. It is convenient to decompose the field of the dipole into spatial-frequency components $\mathrm{k}_{\mathrm{x}}$ and $\mathrm{k}_{\mathrm{y}}(3)$. The full three dimensional (3D) treatment is described in (22). Here we discuss a two-dimensional (2D) case in which the dipole moment is defined per unit 
length as $\mathbf{p}_{2 \mathrm{D}}=\left[\mathrm{p}_{\mathrm{x}}, \mathrm{p}_{\mathrm{z}}\right]$, and the decomposition of its magnetic field can be written as $H_{y}(x, z)=\int H_{y}\left(k_{x}, z\right) e^{i k_{x} x} d k_{x}$, where

$$
H_{y}\left(k_{x}, z\right)=\frac{i \omega}{8 \pi^{2}}\left[p_{z} \frac{k_{x}}{k_{z}} \mp p_{x}\right] e^{i k_{z}|z|},
$$

$\mathrm{k}_{\mathrm{z}}=\left(\mathrm{k}_{0}{ }^{2}-\mathrm{kx}^{2}\right)^{1 / 2}$ is the wavevector along $\mathrm{z}, \omega$ is the angular frequency, $\mathrm{k}_{0}=\omega / \mathrm{c}$, and the phasor notation $\mathbf{H}(\mathbf{r}, \mathrm{t})=\operatorname{Re}\left[\mathbf{H}(\mathbf{r}) \mathrm{e}^{-i \omega t}\right]$ was used. The upper sign in Eq. 1 applies to $\mathrm{z}>$ zdipole while the lower sign applies to $\mathrm{z}<$ zdipole, where zdipole is the dipole coordinate. The $\mathrm{H}_{\mathrm{y}}$ components with $-\mathrm{k}_{0}<\mathrm{k}_{\mathrm{x}}<\mathrm{k}_{0}$ correspond to propagating plane waves forming an angle $\theta=\sin ^{-1}\left(k_{x} / k_{0}\right)$ with the z-axis, while the components with $\left|k_{x}\right|>k_{0}$ are evanescent and present significantly only in the near field (for $\mathrm{z}<<\lambda$ ).

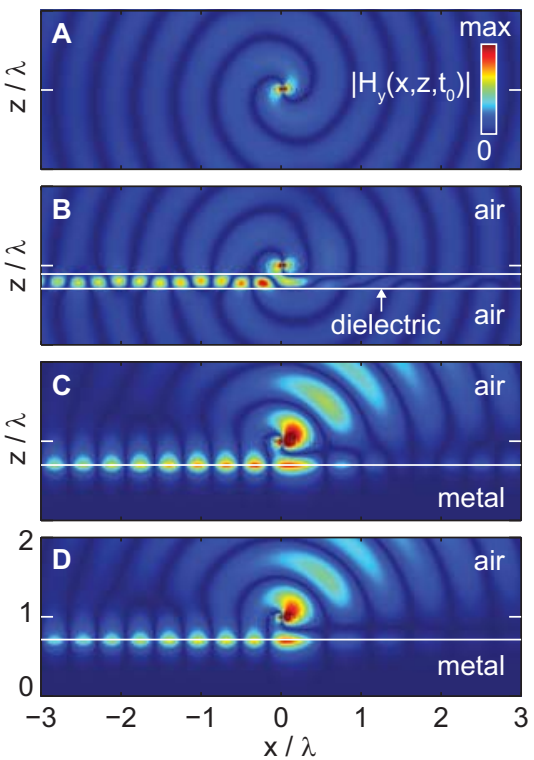

Figure 2. Magnetic field distributions for a $2 \mathrm{D}$ circularly polarized dipole $\mathbf{p}_{2 \mathrm{D}}=[1,-\mathrm{i}](\mathrm{A})$ radiating in free space, $(B)$ exciting a dielectric waveguide mode $(\mathrm{d}=0.1 \lambda, \varepsilon=9+0.1 \mathrm{i}, \mathrm{s}=$ $0.2 \lambda)$, and (C) exciting SPP mode ( $d=0.3 \lambda, \varepsilon=-2+0.1 \mathrm{i}, \mathrm{s}=\infty)$. (D) Same as (C) but with a optimized elliptical dipole $\mathbf{p}_{2 \mathrm{D}}=[1,-0.705 \mathrm{i}]$ to minimize the SPP excitation in the opposite direction. Colour scale is the same for all plots.

The linear superposition of the two orthogonal dipole orientations $p_{x}$ and $p_{z}$ leads to both far-field and near-field interference. The dipole oriented along $\mathbf{z}$ has an odd parity of its spatial frequency spectrum $\mathrm{H}_{\mathrm{y}}\left(\mathrm{k}_{\mathrm{x}}\right)$, while the opposite is true for the $\mathbf{x}$-oriented dipole, which shows an even parity. This suggests that applying the superposition principle to these dipoles could result in a non-symmetric spatial-frequency distribution. The term $\left(\mathrm{k}_{\mathrm{x}} / \mathrm{k}_{\mathrm{z}}\right)$ in Eq. (1) is real valued for propagating components and imaginary for evanescent ones. This means that in order to achieve destructive interference $\left[\mathrm{H}_{\mathrm{y}}\left(\mathrm{k}_{\mathrm{x}}\right)=0\right]$ inside the light cone $\left(\mathrm{k}_{\mathrm{x}}<\mathrm{k}_{0}\right), \mathrm{p}_{\mathrm{x}}$ and $\mathrm{p}_{\mathrm{z}}$ should be in phase (linearly polarized). Remarkably, the interference can take place outside the light cone $\left(\mathrm{k}_{\mathrm{x}}>\mathrm{k}_{0}\right)$ when $\mathrm{p}_{\mathrm{x}}$ and $\mathrm{p}_{z}$ are $90^{\circ}$ out of phase. According to Eq. (1), for a circularly polarized dipole with $\mathbf{p}_{2 \mathrm{D}}=p[1,-i] / \sqrt{2}$, the spectral amplitude of the evanescent components with $\mathrm{k}_{\mathrm{x}}>0$ add up destructively, while for negative $\mathrm{k}_{\mathrm{x}}$ both dipole 
components reinforce each other [Fig. 1(B)]. This near field interference effect is the basis for the selective directional excitation of guided modes by the evanescent components of the dipole field. The directional behaviour that emerges from the evanescent components is a quite counterintuitive result, considering that no preferred direction is observed for the propagating components of the circular dipole, as can be easily shown by noticing that the magnetic field radiated per unit angle, obtained by substituting $k_{x}=k_{0} \sin \theta$ into $H_{y}\left(k_{x}, z\right) d k_{x}$ from Eq. (1), is given by $H_{y}(\theta, z) d \theta=H_{0} e^{\mp i \theta} d \theta$, which has a constant magnitude for all radiating angles $\theta$. From the directional behaviour of the evanescent components, it follows immediately that by placing a structure which supports bound propagating modes with an $\exp \left( \pm i k_{x}^{m} x\right)$ dependence (with $\left.k_{x}^{m}>k_{0}\right)$ in the near field of this dipole, the modes propagating towards negative $\mathbf{x}$ will be dominantly excited over the modes propagating in the positive $\mathbf{x}$ direction with a ratio equal to that of the amplitudes of the corresponding evanescent wave components of the dipole $R=\left|H_{y}\left(k_{x}^{m}\right)\right| /\left|H_{y}\left(-k_{x}^{m}\right)\right|$. The opposite directionality is observed if the direction of rotation of the dipole is reversed. The $z-$ position of the dipole does not affect this ratio, since the evanescent decay, $\exp \left(i k_{z} z\right)$ in Eq. (1), affects the fields with both signs of $k_{x}$ equally.

This effect has been demonstrated numerically for two different waveguides using the spectral decomposition of the dipole and the transfer matrix method (23). The first example is a dielectric slab of thickness $\mathrm{s}=0.2 \lambda$, with permittivity $\varepsilon_{\mathrm{d}}=9+0.1 \mathrm{i}$, placed at a distance $\mathrm{d}=$ $0.1 \lambda$ from the dipole. This slab has a bound waveguided mode that can propagate in opposite directions with the wavevector $\pm k_{x}^{d}= \pm 1.965 k_{0}$ [Fig. 1(B)]. Clearly, the mode with $-k_{x}^{d}$ will be excited by the circular dipole with a much greater amplitude than the one with + $k_{x}^{d^{d}}$ (amplitude ratio $\mathrm{R}=13.4$ ), resulting in unidirectional propagation of the mode within the slab [Fig. 2(B)]. The second example is a semi-infinite metallic medium with permittivity $\varepsilon \mathrm{m}=$ $-2+0.1 \mathrm{i}$, placed at a distance $\mathrm{d}=0.3 \lambda$ from the circular dipole. The air-metal boundary supports counter-propagating SPP modes with wavevectors $\pm k_{x}^{s p p}= \pm 1.41 k_{0}$ [Fig. 1(B)]. Only one of these modes is predominantly excited by the circular dipole (amplitude ratio $\mathrm{R}=$ 5.8), resulting in directional SPP propagation [Fig. 2(C)]. The lobes observed towards the upper right part of the figure correspond to far field radiation from the dipole and its reflection. Animated versions of Figs. 2(A-D) are given in Movies S1-S4.

In both presented examples, a residual non-zero amplitude is still observed for the modes with positive $\mathrm{k}_{\mathrm{x}}$. This residual wave can be eliminated by using a dipole moment with different relative amplitudes in its $\mathrm{x}$ and $\mathrm{z}$ components (elliptical polarization). Relying on Eq. 1, it is possible to design a dipole with a spatial frequency spectrum such that the field is exactly zero for the mode which needs to be inhibited; e.g., $H_{y}\left(k_{x}^{s p p}\right)=0$ for the wavevector of the mode with positive $k_{x}^{s p p}$. This is the case for $\mathbf{p}_{2 \mathrm{D}}=[1,-0.705 \mathrm{i}]$ showing a zero value at exactly the SPP wavevector $k_{x}^{s p p}=+1.41 k_{0}$ [Fig. 1(B)]. The resulting truly unidirectional excitation of SPP wave is shown in Fig. 1(D), with no coupling to the "undesired" back propagating SPP mode. It must be noted that the finite lifetime of the SPPs due to the Ohmic losses causing broadening in the wavevector spectrum may result in some minor spurious excitation.

To experimentally demonstrate the concept, we show the directional excitation of SPPs on a smooth $50 \mathrm{~nm}$ gold film using a symmetric $130 \mathrm{~nm}$ wide slit illuminated with circularly polarized light. The slit plays the role of the 2D circularly polarized dipole, exciting SPPs on the nearby smooth metal film. 3D scenarios may be realized by considering single defects in 
a film (24) or a nanoparticle placed on a metal surface (25). Because the circular polarization of the dipole is required in a plane perpendicular to the metal surface (Fig. 1(A)), the illumination is chosen at a grazing angle along the slit length [Fig. 3(A)]. The leakage radiation of the SPPs into the glass substrate is recorded as bright spots on a CCD camera placed in a spatially filtered Fourier plane (Fig. S1). In the case of linear polarization, both SPPs are excited equally on both sides of the slit, while for left- and right-handed circular polarization, the SPPs are predominantly excited propagating to the right and left side of the slit, respectively [Fig. 3(C)].

A full set of polarization states were studied rotating a $\lambda / 4$ plate in a linearly polarized excitation beam [Fig. 3(D)]. For linear polarization ( $\lambda / 4$ plate: 0,90 and +90 degrees), the expected behaviour of equal excitation of SPPs on both sides of the slit is observed. However, for two circular polarizations of the incident light, SPPs are preferentially excited in one direction. Symmetry requires that the two SPP excitation curves are mirror images of each other, which is clearly observed in the experiment. Small deviations from theoretical predictions obtained for an ideal dipole can be expected to originate from the finite size and shape of the slit, whose dipolar behaviour is not equivalent for fields polarized across the slit and perpendicularly to it. This prevents achieving full directionality and contributes to the observed background intensity [Fig. 3(D)]. A complete control of the polarization ellipse of incoming light $(10,11)$ would optimize the device performance, which following symmetry considerations (22) could potentially achieve a 1:0 contrast ratio. Similarly, directional SPP excitation can be achieved with a slit grating using the same approach (Fig. S2).
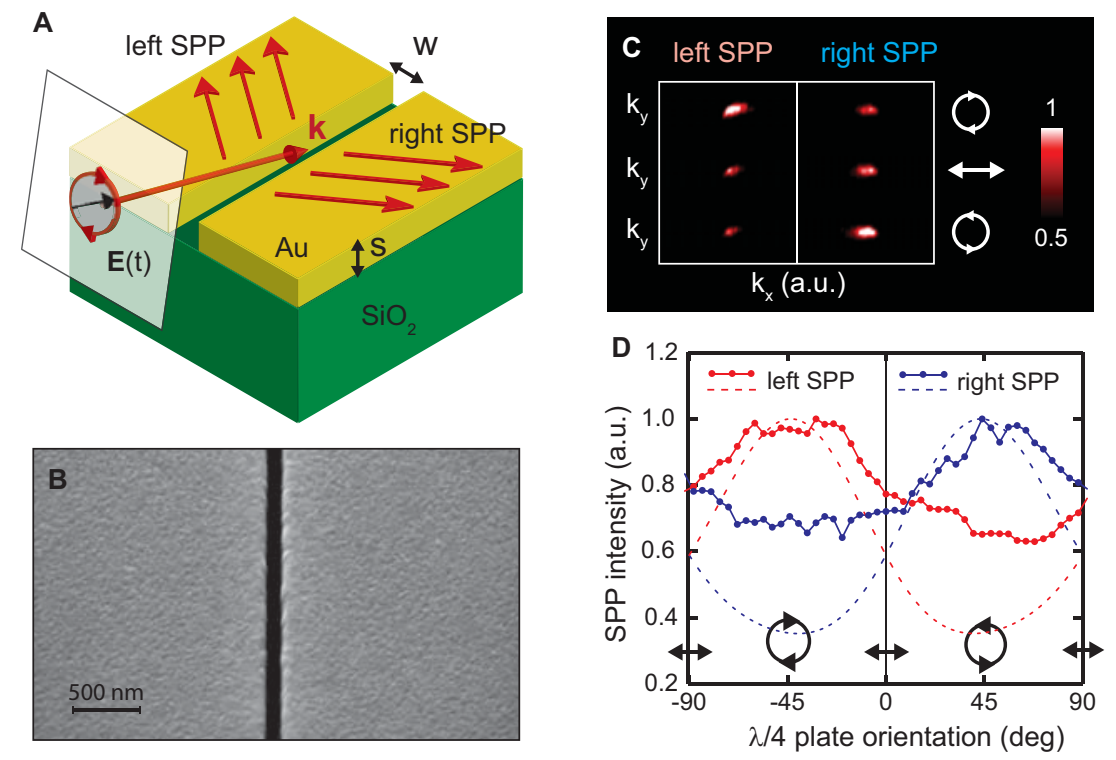

Figure 3. (A) Schematics of the experiment: a plane wave is incident at nearly grazing angle (70 degrees) onto a slit in a metal film. (B) Scanning electron micrograph of the slit. (C) Spatially filtered Fourier plane images of the SPP leakage radiation collected from the sample for different polarization states of the illuminating light. The bright spots correspond to the points on the SPP wavevector arc for the Au/air interface with $\mathrm{ky}_{\mathrm{y}}$ matching the incident wavevector. (D) Experimental (solid lines) and simulated (dashed lines) dependences of the intensity of left and right excited SPPs on the polarization of the illuminating light. 
We have described a fundamental example of vectorial near field interference using a simple circularly or elliptically polarized dipole to achieve directional broadband excitation of guided modes. The underlying physics holds potential and wide applicability to other optical processes. Useful applications may be in the excitation of photonic guided modes exhibiting directional dependence with the direction of the input circularly polarized light, enabling compact nanoscale light sources that could efficiently absorb incoming illumination and couple it to the modes of Si-photonic or plasmonic guides in a desired single direction. It also opens exciting possibilities for directional switching, polarization sorting, and processing of polarization-encoded information, including polarization entangled optical qubits.

Acknowledgements: This work has been supported in part by EPSRC (grant EP/H000917/2). F. J. Rodríguez-Fortuño acknowledges support from grant FPI of GV. A. Martínez acknowledges financial support from Spanish government (contracts Consolider EMET CSD2008-00066 and TEC2011-28664-C02-02). P. Ginzburg acknowledges the Royal Society for a Newton International Fellowship.

\section{References and Notes}

1. N. Yu et al., Science 334, 333-337 (2011).

2. X. Ni, N. K. Emani, A. V Kildishev, A. Boltasseva, V. M. Shalaev, Science 335, 427 (2012).

3. L. Novotny, B. Hecht, Principles of Nano-Optics (Cambridge University Press, 2011).

4. R. Merlin, Science 317, 927-929 (2007).

5. J. B. Pendry, Phys. Rev. Lett. 85, 3966-3969 (2000).

6. L. E. Helseth, Opt. Commun. 281, 1981-1985 (2008).

7. G. V. Eleftheriades, A. M. H. Wong, IEEE Microw. Wirel. Co. 18, 236-238 (2008).

8. J. Y. Lee et al., Nature 460, 498-501 (2009).

9. M. Stockman, S. Faleev, D. Bergman, Phys. Rev. Lett. 88, 067402 (2002).

10. M. Aeschlimann et al., New Journal of Physics 14, 033030 (2012).

11. M. Sukharev, T. Seideman, Nano Lett. 6, 715-719 (2006).

12. W. L. Barnes, A. Dereux, T. W. Ebbesen, Nature 424, 824-830 (2003).

13. J. a Schuller et al., Nat. Mater. 9, 193-204 (2010).

14. H. Kim, B. Lee, Plasmonics 4, 153-159 (2009).

15. N. Bonod, E. Popov, L. Li, B. Chernov, Opt. Express 15, 11427-11432 (2007).

16. J.-S. Bouillard, S. Vilain, W. Dickson, G. A. Wurtz, A. V Zayats, Scientific reports 2, 829 (2012).

17. X. Li, Q. Tan, B. Bai, G. Jin, Appl. Phys. Lett. 98, 251109 (2011).

18. I. P. Radko et al., Opt. Express 17, 7228-7232 (2009).

19. Y. Liu et al., Nano Lett. 12, 4853-4858 (2012).

20. A. G. Curto et al., Science 329, 930-933 (2010). 
21. G. Roelkens et al., J. Nanosci. Nanotechnol. 10, 1551-1562 (2010).

22. See Supplemental Material at http://www.sciencemag.org/content/340/6130/328/suppl/DC1 for Movies S1-S4, materials and methods, and supplementary text.

23. W. N. Hansen, J. Opt. Soc. Am. 58, 380-388 (1968).

24. N. Rotenberg et al., Phys. Rev. Lett. 108, 127402 (2012).

25. Z. Ruan, S. Fan, Phys. Rev. Lett. 105, 013901 (2010). 